\title{
DECONSTRUCTION-RECONSTRUCTION OF COMPUTER GAME AND IMMERSIVE ENVIRONMENT SEMIOTICS
}

\author{
Colin B. Price, June S. Moore, Joanne M. Kuzma \\ Computing at the Worcester Business School \\ University of Worcester \\ Henwick Grove, Worcester, UK \\ c.price@worc.ac.uk \\ http://www.worc.ac.uk/departs/bm it/colin/index.htm
}

\begin{abstract}
Computer game technology produces compelling 'immersive environments' where our digitally-native youth play and explore. Players absorb visual, auditory and other signs and process these in real time, making rapid choices on how to move through the game-space to experience 'meaningful play'. How can immersive environments be designed to elicit perception and understanding of signs? In this paper we explore game design and gameplay from a semiotic perspective, focusing on the creation of meaning for players as they play the game. We propose a theory of game design based on semiotics.
\end{abstract}

\section{INTRODUCTION}

We have all played games according to rules, in a socio-cultural space; marbles in the school playground, playing chess or monopoly where the space was convivial but the rules were motivating. Today the playground remains; there are no marbles, there are 'gogos'. But the rules remain and are accepted within the play systems of our youth. Outside the playground our youth is playing another sort of game which is establishing a new culture, that of the 'Digital Natives'. This culture has transposed space from the outdoor (UK) seasonal climate into an indoor space of human interaction with sociocollaborative opportunities, thanks to the affordances of computer technology. Previous generations of our youth have engaged with organizations such as the UK 'scouts' or 'brownies'. Our Digital Natives aspire to engage with similar active communities, but these are 'on-line' or 'virtual'. What is going on here? To understand this, we must consider the ontology of computer games and the design processes of those who provide this. We propose that the design of computer games and the ensuing game play should be viewed from several perspectives. The first is 'games as semiology'; in game play players actively construct meaning. The second is 'games as rules', (following traditional game design wisdom), informing the player how to react in the social context of the game. The third is the 'game mechanics', technical game affordances, such as textures, objects, characters.

This paper starts from the semiotic perspective and considers how and why players play games (The semiotics of computer games). A deconstruction of game rules leads to the thesis that 'classical' game rules (such as Scrabble or Monopoly) when transferred to computer games, become identified with space (The ontology of computer games). A short consideration on the use of the image is given in The place of the image. In 
Measurement of game-play semiotics we present results of research on how signs within various games influenced player choice. A theory of computer game design based on our findings is presented in Towards a theory of game-play design. One immersive environment we have developed based on our theory, for Primary School Mathematics teaching, is discussed in the Case Study. This paper is accompanied by associated webpages providing example resources [1].

\section{THE SEMIOTICS OF COMPUTER GAMES}

People play computer games for a reason. People watch movies for a reason. But these reasons are not the same, even though both media are immersive and engulfing. By 'reason' we mean a human response to a cognitive, social or individual engagement with a medium. Computer games have been tagged as 'immersive', 'interactional' where 'interaction' has been associated with 'engagement'. Yet the reason for this evident engagement must be understood. Salen and Zimmerman identify the viability of computer games as following from their ability to provide 'meaningful play' [2]. This means that the player's actions produce effects which create meaning, e.g., when a player activates a switch there is a discernible result; the door opens. The action also acquires meaning if the action has an effect at a later time in the game; picking up a weapon may slow you down now but it allows you to shoot the monster when it appears later. To create meaningful events and play, the designer should pay attention to the field of semiotics, the study of meaning.

Semiotics emerged from the studies of the linguist de Saussure and fed rapidly into other disciplines such as the anthropology of Levi-Strauss, the social mythology of Barthes, and the philosophy of Derrida. Semiotics concerns how meanings are made based on the concept of a sign (or signifier) and the signified, the object or idea to which it points. In the above example the switch is the sign and the signified is "opening". Game semiotics has to be considered from the perspective of the level-designer as well as the player. Currently, game design can be seen as a process of "world building" which Holtzman describes as a process of how using "a system of symbols to represent a view of reality, the artist shares his consciousness of aspects of that reality" [3]. In a 3D computer game such as Half-Life or Unreal Tournament these signs are provided by static meshes, textures, sound, music, animations and other assets. Yet their use alone does not establish a semiotic system since it is the player who processes these signs and produces meaning which may extend beyond the artist's project. Also, while these signs represent the world to the player (and refer to signifieds in the real world), they gain their semiotic value from their inter-relationships. This establishes a semiotic system as the player plays the game.

A useful definition of semiotics may be found in the work of the American semiotician C.S. Peirce. He defines a sign as "something that stands for something, to somebody in some respect or capacity". There are four factors which describe a sign, these are (i) A sign represents something which is not itself, (ii) A sign must be interpreted, (iii) When a sign is interpreted then meaning is generated, (iv) Interpretation is shaped by context. One of de Saussure's fundamental insights was that signs carry no absolute meaning, but that meaning is interpreted within a cultural 
context, such as game experience. When we come across a switch in a computer game and do not know its purpose, flipping the switch once has an immediate effect which produces meaning. It is the job of the level designer to ensure that the sign is placed within a context or semiotic system. This links into the notion of 'game rules' which inform viable game play, like the rules of grammar in language. The impact of the cultural context becomes clear when we observe the game-play of our 'Digital Natives' (those born into a digital world) compared to 'Digital Immigrants' (those who have to learn this world). These native game players expect a semiotic system and instinctively hunt out the signs located in the game.

In computer game design, conventions for the use of signs are beginning to emerge. Spatial cues may be placed to direct the player; non-player characters may be deployed to engage the player providing e.g. instruction; lighting and sounds provide attraction and establish the tempo of game play.

The semiotics of computer games has been studied in detail by David Myers [4]. He points out that game play can be seen as a sequence of significations responding to encountered signs, but where there is a clear relationship between these signs. As a player moves through a level, the experience of encountering a succession of signs creates a context in which the next sign can be interpreted. This process of recursive contextualization creates meaning and also grounds the notion of 'interactivity' within computer games. A comparison with literature is useful. Here the semiotic process involves a communication of meanings and values through a common language. Computer games do not have this language; game play is a process of discovering meaning which is not expressed in a language based on a grammar, but through a semiotic system. To some extent this liberates the player from the motivating (but restricting) literary experience.

Myers makes an interesting comparison of two 'game genres'. In 'action' games (such as Doom or Unreal Tournament 'shoot 'em up') the signs are first-order or denotative. Value and meaning are given to these signs by a direct phenomenological experience of these signs and the patterns which emerge through reading these signs. On the other hand, in 'adventure' games such as Zork or Myst, the signification is connotative or second-order. In these games the player is expected to construct meaningful play by experiencing a stream of information, often presented as textual elements, and to assimilate each sign into a story. This requires much more player effort. Action games provide oppositional relationships between signs (the player kills the monster or gets killed) while adventure games provide contextual relationships between signs (the player uncovers text fragments which provide the game story). This recursive (and therefore infinite) semiotic process is a good model of how we make sense of the real world when interacting with cultural-specific signs. This aligns with the constructivist understanding of perception which asserts that the concept of an objective representation of reality is an illusion. All we do in the real world is to execute a continuous process of making meaning. Perhaps the real world is, therefore, virtual? 


\section{THE ONTOLOGY OF COMPUTER GAMES}

In a 'classical game' such as Scrabble or Monopoly, the 'rules' of the game are printed on the box. Computer game players eschew this box, and rush into the game to discover the rules. Their rules are not explicit; the gamer expects to discover the rules during play. This is especially true of 'action' games rather than 'adventure' games, where rules may often have to be stated. The game designer conceives the rules as a set of player actions, and contextualises these within space [5]. We propose that the rules of classical games are embodied in the space of computer games, which therefore forms a part of its ontology. Yet this is not an architectural space but a space where the player's actions take place. The design of such a space may be called 'design by verb' which is akin to the architects' manifesto 'form follows function'.

Having established that space is a component of the computer game ontology it remains to consider how to extend such a framework to elicit 'meaningful play'. Space has been addressed by many; from Klee's The Thinking Eye [6] to Kevin Lynch's five elements of urban planning [7], pace, Einstein. Both Klee and Lynch imply a strata of composition, where 'points', 'lines', 'planes', or 'paths', 'edges', 'districts', 'nodes', and 'landmarks' overlay and interact. Such strata have been formally employed by the architect Bernard Tschumi in his design of La Villette (Paris) [8]. Lynch suggests that urban designers should consider the narrative potential of geographical space. He describes city planning as "the deliberate manipulation of the world for sensuous ends". So he creates a link between location and narrative. This is also true of La Villette where performances are located in specific areas. Each stratum contains, but between the strata informs. These spatial strata may be usefully overlaid to create a gameplay semiosis. In our theory presented in the Finale we assert that the ontology of computer games is grounded in space. It is within this grounding that other considerations such as 'game narrative', or principles of game design such as 'non-linearity', 'risk', 'goals', or 'tunnelling' should be situated. The technological affordances of the computer game engine (movers, pickups, NPCs) also have to be situated in space. But the final stratum of this ontology is the semiotic level related to space. Our thesis is therefore that computer game ontology consists of strata of geographical space including technical elements of game design, semiotics and perhaps narrative.

To some extent this space shares some characteristics with architectural space. In architecture, 'player' movement is called circulation which is defined by form and space, and to some extent lighting. Computer games also use form and space to define circulation, but make more use of images, music, sound and interactions to guide the player through the underlying semiotic system. Computer game design uses a particular form of circulation called 'tunnelling' where the player is led through a series of subgoals (e.g. challenges) on the way to the final goal (resolution of conflict). We interpret this as engaging with a series of semiotic sub-systems in which the player has to induce meaning in order to progress. Architects create places within space which are pleasant and comfortable. Game designers create places of danger and risk in which the player gains pleasure by overcoming these. 
Our suggestion that space is a fundamental component of the ontology, without any mention of time seems strange in this post-Einsteinian epoch, until we remember the nature of game time. Games are played with a single time-scale, action games run at full speed with little opportunity to pause (except perhaps during cut-scenes). A player who pauses will get shot. Movies make use of variable time-scale (pace), fast action sequences are interspersed with moments of discussion. A movie-goer would not tolerate an action movie running from start to end at full speed. One way of creating a perception of a varied time-scale is the use of the 'dramatic arc'. Here a tension (between conflict and resolution) arises according to the player's uncertainty about the outcome. Tension at the start of play is low where uncertainty is high. As the game is played this tension rises until the outcome seems inevitable when the tension falls. The player's perception of time tracks this level of dramatic tension. Perceived time may also be directly manipulated by the game designer through a change in the density of the signs located in the game. Space does not allow for a full discussion of time within computer games, though we have already alluded to this issue. It has been suggested that the study of cybernetic systems may be useful in adapting gameplay over time [9]. A simple example would be the use of negative feedback in reducing the strength of the opponent when the player is slaughtered too often. We suggest a more radical approach; the use of the theory of self-organizing dynamic systems where the designer crafts systems of objects and interactions to allow the expression of an emergent dynamic behaviour. This will be the subject of an imminent publication.

The power of computer games to engage the player is often attributed to their high degree of interactivity. While this may seem obvious, there is debate in the literature on the meaning of the word interactive. Within our ontology, interactivity emerges as a consequence of the player reading signs and responding to the signifieds. There is a two-way flow of information from the game to the player and from the player into the game, In this way interactivity is a consequence of the process of recursive contextualisation as mentioned above. It is as much to do with this process as with the mouse and keyboard (which have a limited input value).

\section{THE PLACE OF THE IMAGE}

As the scientific understanding of the human mind and the human visual system has progressed, so has our understanding of the meaning of the image. No longer is an image a pictorial or abstract representation of an object. Images have become a process, a part of our daily activities and culture. Moreover, through digital technology they have become an interface between humans and computers which bind that technology to being a human in context nouveux. How has the computer game industry engaged with the image? The historical thrust of the industry has to improve hardware and software to strive for increasing levels of realism. Yet high-fidelity realism can detract from player enjoyment. Crawford suggests that enhanced realism may impede communication [10]. Perhaps the player feels insufficiently removed from reality to be motivated to play? Or perhaps realism detracts from a semiotic engagement; an inability to read the signs? Here it is important to understand how Digital Natives interact with image-spaces. Burnett notes, "The digital revolution is altering the fabric of research and practice in 
the sciences, arts and engineering and challenging many conventional wisdoms about the seemingly transparent relationships between images and meaning, mind and thought, as well as culture and identity" [11]. Images no longer need captions to explain, they explain themselves. The communication primacy, traditionally associated to written (or spoken language), and its linguistic baggage, has been overcome. Grammar-less images communicate directly. While there is a resulting potential desire to assert the primacy of image over text, this desire must be suppressed, since alternative modalities such as sound and music will then be in turn neglected. A multimodal semiotics must be embraced. Full circle: why neglect the textual component of communication in computer games?

\section{MEASUREMENT OF GAME-PLAY SEMIOTICS}

Research was performed into how players moved through game levels which included signs such as visuals, text and audio (sounds and music). Some 20 students at Worcester University collaborated in this project. We augmented several Unreal Tournament, (UT2004) game levels with invisible 'locators' programmed to record players' location in the game, including a time-stamp, and a screen-shot. We also designed specific levels to factor out semiotic elements such as visuals and audio signs, and to explore inter-relations between these.

There were three phases to these investigations; movement through (i) existing UT2004 game levels, (ii) game levels we designed using an external environment (iii) game levels we designed depicting an internal 'labyrinthine' environment. The results of (i) were used to inform the design of (ii) and (iii).

The analysis of data from augmented UT2004 game levels revealed some strong behavioural factors: (i) faced with a left-right choice of movement, most players chose left, (ii) faced with an up-down choice most players chose down, (iii) players were influenced by animated rather than static visuals such as 'adrenaline capsules', (iv) players followed explicit dynamic 'arrow signs', (v) in external 'terrain' levels, players tended to run through valleys rather than climb mountains, (vi) players responded highly to non-player characters (and chose to shoot them). These identified factors above were used to create levels where factors were separated ensuring that the synthetic levels did not contain any extraneous signs. These levels were based on a common external environment based on a Japanese Temple theme (figure 1). 


\section{EVA 2009 London Conference 6-8 July \\ Colin B. Price et al.}
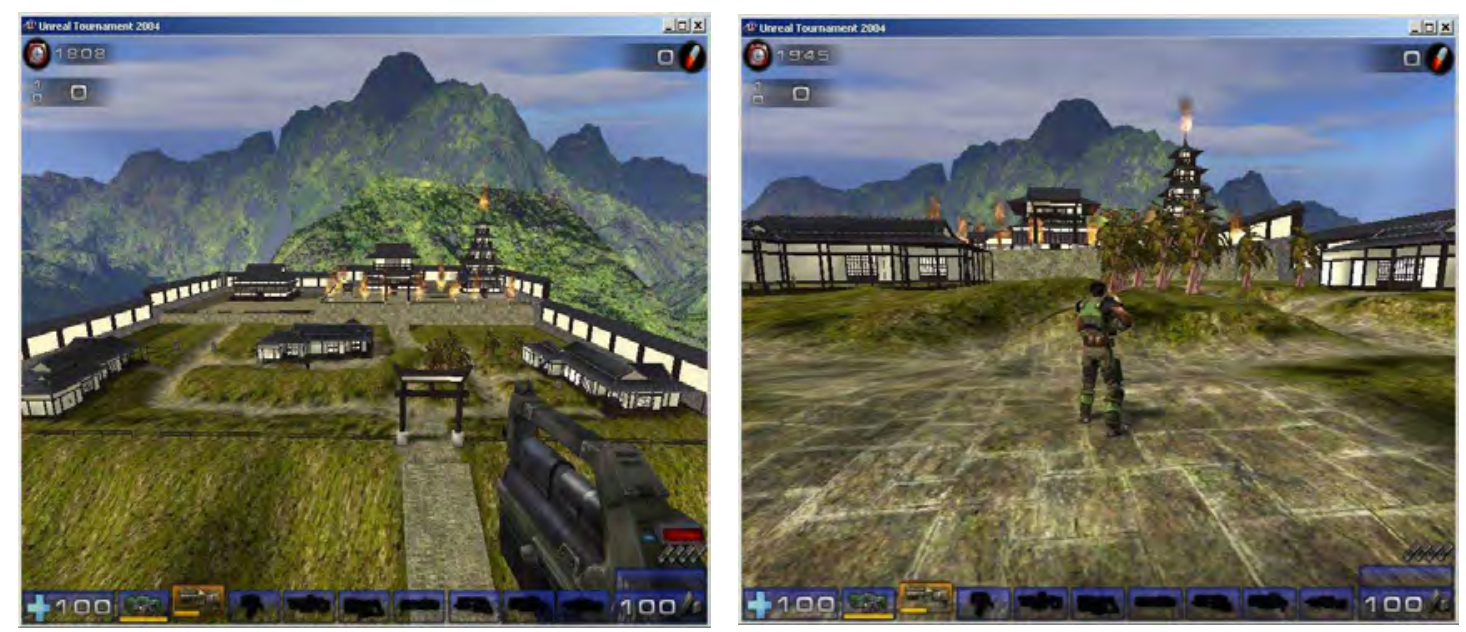

Figure 1. Left shows aerial overview of the 'Fires' Japanese Temple immersive environment. Right shows the third-person viewpoint of the player

This aerial view shows a division into two spaces. The lower space is a landscape with grass, paths and trees. There are three buildings and a Japanese 'gate' providing a clear entry point. The player starts the game below this entry point. The upper space is flat and is divided into two areas containing buildings and a temple. A number of variant levels were investigated based on this common spatial map. Each variant map was supplemented with different semiotic elements. These were: (i) Localised fires which were intended to attract the player, located at points around the temple, (ii) Generalized fires located at the top-right temple area, (iii) Deployment of objects to attract the player, (iv) Three levels where trees were presented to guide the player, (v) Iconic semiotics involving signs such as 'danger do not enter' or dynamic arrows designed to influence movement, (vi) Objects situated in areas within the level.

Analysis of player movement through these levels revealed several general patterns: (i) players were attracted to dynamic textures (such as fires), and some players chose to explore, (ii) players were not attracted to static objects, such as clusters of barrels or a whisky bottle, (iii) players were not influenced by the spatial structures provided by avenues of trees, (iv) players responded well to the dynamic arrows. Interestingly, the analysis showed a significant difference in player movement based on their previous experience with computer games. Game-players exhibited a more structured approach to movement, whereas game novices showed a more random or experiential approach (figure 2). 


\section{EVA 2009 London Conference 6-8 July \\ Colin B. Price et al.}
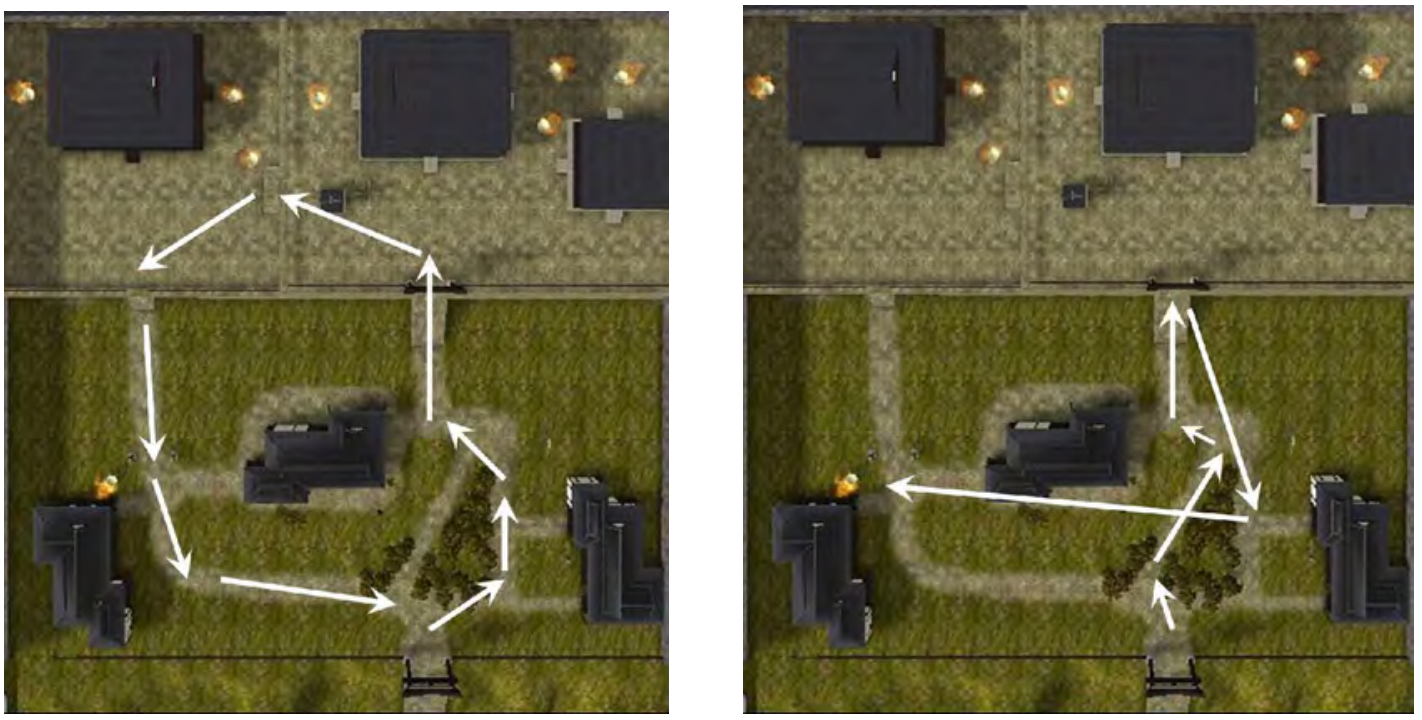

Figure 2. Arrows show the circulation of two players through the "Fire" level. The experienced game player (left) makes a systematic exploration of the level while the novice player (right) is immediately attracted to the fire signs.

Players tended to read signs placed in the far field (such as the fires). Once moving they were reluctant to change direction; the signs presented by near-field visuals rushed by like countryside viewed from within a train. Even though the player may think they are moving through the immersive environment, their perception may be one of the environment flowing by. This could explain why players were not attracted by the avenue of trees. Static objects or objects with static textures tended not to attract the player; only dynamic objects or textures worked as signs. In the image-rich immersive environment they required a difference to be able to recognize a sign. Analysis of these observations informed the construction of the 'Labyrinthine' levels where objects, lighting and audios were introduced. Labyrinths started on the drawing-board as an $8 \times 8$ matrix of interconnected rooms. Interconnections were removed randomly with a change of $1 / 3$. The design of these levels was quite sophisticated involving choices of visuals, objects and sounds. Sounds were associated with rooms (moving into a dark room the player heard a scream, moving into a bright room the player heard laughter). One level labelled each room with Cartesian coordinates, again signs whose meaning had to be discovered. These levels were then offered to players. At the time of writing the primary data is coming in and we look forward to sharing our analysis on the associated web-pages.

\section{TOWARDS A THEORY OF GAME DESIGN}

The starting point for any theory has to be an accepted game ontology, which we suggest starts with space as discussed above. Time is bracketed (for the moment). 

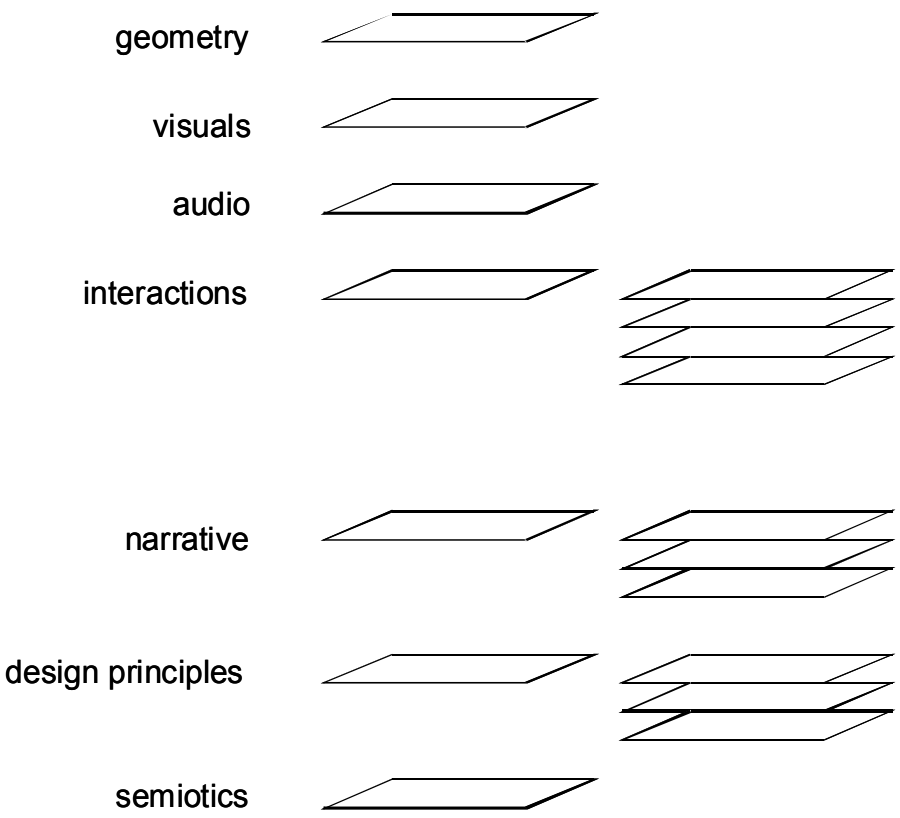

Figure 3. Our proposed computer game ontology: From the top down, four strata of game technology, a narrative stratum, a stratum of computer game design principles and the semiotics stratum. Sub-strata are indicated on the right.

Taking the lead from architects and planners such as Lynch and Tschumi, we propose a number of strata which embody game-design principles, approaches to narrative, and the technological affordances of the game engine. Other strata include game design principles and, of course semiotics. This is illustrated in figure 3 . There are a number of primary strata: (i) Geometry, (ii) Visuals, (iii) Audio, (iv) Interactions. These refer to the technological affordances of the game engine. Then we have (v) Narrative, (vi) Game design principles, (vii) Semiotics. Various strata have sub-strata. Each stratum is a 2D geographical map of where elements in that stratum are located within the game.

We suggest that the game design process will involve a simultaneous consideration of all strata, e.g., the location of a sound and the associated visual (bird on a tree) is linked to the local geometry (hillside) and the sign (entering a pleasant area devoid of opposition). This point-location must be brought into association with other geometrical elements, e.g. a line (path along the hillside) and hence into association with the game design (tunnelling through challenges). The line then becomes a sign for challenges. This suggests that, to achieve a consistent semiotic system, all lines would have to be associated with challenges. The semiotic stratum becomes an organizer to provide a game geometry which will support meaningful play. 


\section{CASE STUDY - PRIMARY SCHOOL MATHEMATICS}

The results of the analysis presented above and subsequent theory has been applied to the design of an educational immersive environment to support the learning of mathematics within the UK Primary School system. We have worked for some time with this school producing educational immersive environments. Our approach is iterative, where teachers critically analyse a series of prototypes which converged to their needs. At each stage of the educational immersive environment design process, the strata and their vertical inter-relationships were considered. The semiotic stratum was taken as a focus, to coordinate the other elements of design. The context is a cartoon world where the learner discovers various 'learning areas', 'play areas', 'danger areas' which are indicated by various signs. Through experiencing denotative signs, the learner soon constructs meaningful 'play'. The educational immersive environment is shown in figure 4.
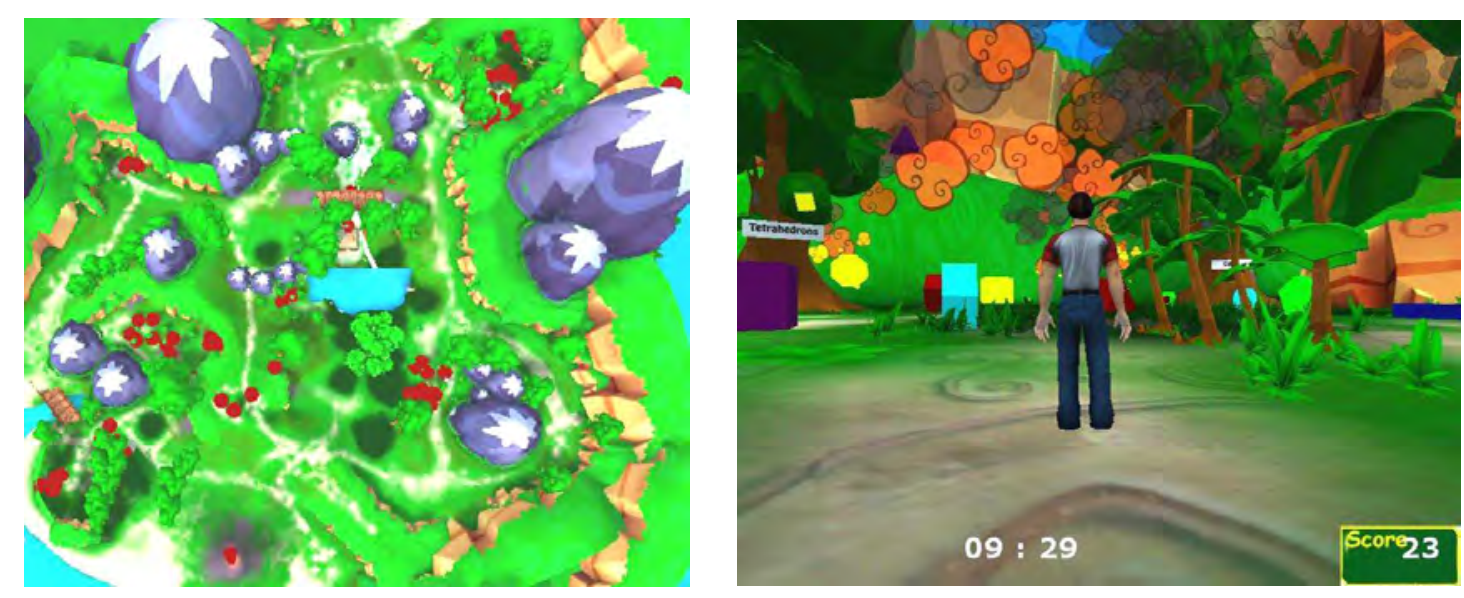

Figure 4. An educational immersive environment for Primary Maths education: Left shows an aerial view showing areas connected by paths situated in a terrain. Right shows a learning area showing consistent textures used on trees, smoke and on the terrain.

The semiotic stratum has been realised in a number of ways. First, all textures have been chosen with a theme of a spiral motif. This is applied to the ground, hills, trees and fires to produce a consistent visual experience with no oppositions. The learning areas are identified by the audio sign of the Mission Impossible tune, play areas are signed the tune from the Magic Roundabout and forbidden areas are signed by the sound of howling wolves. Connections between areas are signed by the use of worn-down paths (in a contrasting colour). All of these signs induce meaning when they are first experienced. A series of huts is used to teleport (rapidly transfer) the learner between various areas. These huts act as signs for the signified teleportation, again their meaning is learned through experience. The learners discover that trees which start to grow signify the correct solution to a problem. This high level of semiotic design has produced an educational immersive environment which the learners can first genuinely 
explore to expose the 'game rules', and then to use these in a process of enhancing their math skills. But of course, both processes of exploration (fun!) and learning (fun?) occur together. The learner is motivated through a realisation that the educational immersive environment contains many signs and signifieds which are there to be discovered. It is clear that a correctly designed game ontology based on a strong semiotic system is able to motivate the learner and to provide fun implicitly, without resorting to an artificial 'fun' design element.

\section{FINALE : A GENERATIVE SEMIOTIC SYSTEM}

While playing a computer game, the player moves relentlessly through the game level engaging with an underlying semiotic system without realising it exists. The player experiences a phenomenological momentum: go forward means go forward... 'go forward!'. Some visuals 'flow by'; others are seen and interpreted as signs. These signs generate meaning and so inform the next action, 'What to do next?' Meaningful play ensues. On the one hand the game designer develops the semiotic game stratum, on the other, in game-play, this is deconstructed by the player who must learn the semiotics step by step, and perhaps even challenge it (through the use of 'cheats'). Reconstruction is obtained when the player has learned the rules and feels confident to play the game for its own sake. The semiotic system is realised. Meaningful play ensues. The player has fun. Enormous and significant fun!

\section{ACKNOWLEDGEMENTS}

We would like to acknowledge the work of our second-year student Neil Sherwood for allowing us to adapt his Japanese Temple game. We also thank the creator of the basic assets used in our mathematics educational immersive environment, Mathieu Grangon. Thanks also to the Headmaster, teachers and pupils of Cherry Orchard Primary School (Worcester, UK) for actively supporting our work.

\section{References}

[1] Supporting web-pages at: http://www.worc.ac.uk/departs/bm_it/colin/Resources/ EVA2009/index.htm

[2] SALEN, S. and ZIMMERMAN, E. Rules of play. MIT Press, 2000.

[3] HOLTZMAN, S. Digital mantras : the languages of abstract and virtual worlds. MIT Press, 1994.

[4] MYERS, D. The nature of computer games. Peter Lang, 2003.

[5] TOTTEN, C,W. Game design and architecture. Master of Architecture thesis. Washington DC : Catholic University of America, 2008.

[6] KLEE, P. The thinking eye. London : Percy Lund, 1961.

[7] LYNCH, K. The image of the city. London : MIT Press, 1960.

[8] TSCHUMI, B. http://www.tschumi.com/

[9] SALEN, S and ZIMMERMAN, E. Rules of play. MIT Press, 2000.

[10] CRAWFORD, C. Chris Crawford on game design. New Riders, 2003.

[11] BURNETT, R. How images think. MIT Press, 2005. p.xv 